\title{
Acceptability and Effectiveness of Linking HIV-Positive Family Planning Clients to Treatment and Care Services among Health Care Providers in Kenya
}

\author{
Wilson Liambila ${ }^{1 *}$, Francis Obare ${ }^{1}$, Harriet Birungi ${ }^{1}$, Shiphrah Njeri Kuria ${ }^{2}$, Ruth Wayua Muia ${ }^{2}$, Christine Awuor ${ }^{3}$, Joyce W Maina ${ }^{4}$ and Mary \\ N Maina ${ }^{4}$
}

${ }^{1}$ Population Council-Nairobi Office, Kenya

${ }^{2}$ Ministry of Health - Division of Reproductive Health (DRH), Kenya

${ }^{3}$ Ministry of Health - National AIDS and STI Control program (NASCOP), Kenya

${ }^{4}$ District Health Management Teams- Nyeri and Kiambu Counties of Central Region -Kenya

\begin{abstract}
Although there has been growing interest in the provision of integrated reproductive health and HIV services in sub-Saharan Africa in recent decades, there is limited evidence on effectiveness of linking family planning clients who test HIV-positive to comprehensive care centers with respect to such outcomes as uptake of care and support services as well as initiation of clients to antiretroviral therapy for those eligible. This paper examined the acceptability and effectiveness of linking HIV-positive family planning (FP) clients to treatment and care services among health care providers in Kenya. Data are from a pre- and post-intervention study without a comparison group. Intervention activities included modifying the referral system, reviewing and updating training materials and job aids, training service providers on the updated tools, and conducting support supervision. Data collection involved facility assessment, provider interviews, and record reviews. Analysis entails descriptive statistics including frequencies, percentages and scores with significant tests of means and proportions to determine if there were any significant differences between baseline and endline. The results show that although most facilities were not adequately prepared in terms of infrastructure to support linking HIV-positive family planning clients to treatment and care services, there was significant improvement in provider acceptance of referral practices after than before the interventions. For example, the proportion of providers who used a directory for referral rose from $8 \%$ at baseline to $58 \%$ at endline $(p<0.01)$ while the proportion of providers who discussed referral of HIV-positive FP clients improved from $71 \%$ at baseline to $96 \%$ at the endline $(p<0.05)$. In addition, whereas there was initially no referral of HIVpositive FP clients to comprehensive care centers (CCCs), most of the HIV-positive FP clients received at CCCs $(93 \%)$ were from within the Maternal-Child-Health $(\mathrm{MCH})$ /FP unit of the same facility during the intervention period indicating that the referral system was effective.
\end{abstract}

Keywords: Integrated services; Family planning and HIV/AIDS; Referral services; Treatment and care; Kenya

\section{Introduction}

In recent decades, the provision of integrated reproductive health (RH) and HIV services in sub-Saharan Africa has generated a lot of interest from researchers, program implementers and policy makers with arguments for and against integration. Integration in this context refers to the delivery of the two sets of services within the same setting, during the same hours, and, preferably, under the same roof, or as part of a facilitated referral within the same facility or to off-site facilities $[1,2]$. Thus, clients or patients typically receive both $\mathrm{RH}$ and HIV services in one visit $[1,2]$. The Joint United Nations Programme on HIV/AIDS (UNAIDS), on the other hand, defines integration as 'joining together different kinds of services or operational programs in order to maximize outcomes, e.g., by organizing referrals from one service to another or offering one-stop comprehensive and integrated services' [3].

The arguments for integrating RH-HIV services include the fact that the risk factors for HIV/sexually transmitted infections (STIs) and unintended pregnancies are similar and that the approach could enhance efficiency in the delivery of both services since they require similar inputs such as skills, equipment and supplies. In addition, integrating RH-HIV services is perceived to enhance access to quality of care and to address the needs of those clients that demand both services [4]. On the other hand, arguments against integration are premised on the ground that it would affect the quality of the services offered since the two types of services have different structures [5].

Despite the arguments for and against integration, it is worth noting that for women with HIV who do not wish to become pregnant, family planning is a proven, cost-effective strategy for preventing motherto-child transmission of HIV (PMTCT) and, therefore, reducing the number of children needing HIV treatment, care and support [6-10]. Moreover, the interconnectedness of family planning and HIV/AIDS needs has long been recognized in major international declarations. At the 1994 International Conference on Population and Development (ICPD), both family planning and HIV services were deemed critical to achieving sexual and reproductive health goals.

The ICPD plan of action called for integrated services that addressed

*Corresponding author: Wilson Liambila, Population Council-Nairobi Office, Nairobi, Kenya, Tel: 254-2-2713480; Fax: 254-2-2713479; E-mail: wliambila@popcouncil.org

Received June 26, 2013; Accepted August 20, 2013; Published August 25, 2013

Citation: Liambila W, Obare F, Birungi H, Kuria SN, Muia RW, et al. (2013) Acceptability and Effectiveness of Linking HIV-Positive Family Planning Clients to Treatment and Care Services among Health Care Providers in Kenya. J AIDS Clin Res 4: 232. doi:10.4172/2155-6113.1000232

Copyright: @ 2013 Liambila W, et al. This is an open-access article distributed under the terms of the Creative Commons Attribution License, which permits unrestricted use, distribution, and reproduction in any medium, provided the original author and source are credited. 
the sexual and reproductive health needs of individuals in a holistic manner [11,12]. Although there are established connections between family planning and HIV, the two types of reproductive health needs are not effectively bridged in practice. For example, rates of unintended pregnancies remain alarmingly high in women with HIV, and family planning interventions have been underutilized in HIV prevention, care and treatment programs [13]. In addition, HIV programs have emerged primarily as separate 'silos' and only minimal efforts have been made to leverage and integrate them with existing family planning infrastructures [14].

Integrated sexual and reproductive health (SRH)-HIV services reach people or individuals in need of these services in a variety of ways. Examples of these approaches or types of integration include on-site, off-site and mixed types [4]. The on-site approach could be a one-stop shop where sexual and reproductive health (SRH)-HIV integrated services are offered by one service provider in one room during the same consultation [4]. It could also be a one-shop approach or supermarket approach where more than one service provider within one facility offers the services during the same visit [4]. In addition, internal referral could take place within the same facility. The off-site approach is where a client accesses one type of SRH-HIV integrated service and receives the other service outside the facility or site through a referral [4]. The mixed-model approach, on the other hand, is where the base or index service is provided at the facility or site, but the other service is obtained through facilitated referral from another facility or facilities mainly due to inadequacies in skills, equipment or supplies in the facilities making the referrals [4].

A recent study that examined the evidence of the impact of integrating any component of STI or HIV prevention, care, and treatment into a family planning setting in developing countries concluded that integrated services can have a positive impact on client satisfaction, improve access to respective services, reduce clinic-based HIV-related stigma, and that they are cost-effective [15]. However, there was inconclusive evidence of FP services reaching men and adolescents [15]. In addition, providers frequently missed opportunities to integrate care awhile the capacity to maintain quality of care was also influenced by many programmatic challenges [15]. The authors concluded that in order to achieve effective RH-HIV integration, health managers need to determine appropriate service delivery models based on a consideration of epidemiological, structural, and health-systems factors [15]. Further review of literature indicates that there exists ample evidence, which justifies the need to combine reproductive health services (of which family planning activities are part of) with HIV services and that integrating HIV into RH services improves access to care and reduces clinic-based HIV-related stigma [16-18].

Efforts to integrate RH services in Kenya began after the 1994 ICPD with a focus on integrating STIs into RH, HIV into FP and FP into HIV services $[4,19]$. The implementation of RH-HIV integrated services is carried out in accordance with the way Kenya's health care system, whose structure is based on six levels of delivering essential packages for health care. The levels are community, dispensary, health centre, district hospital, provincial hospital and the national referral hospitals. Each of these levels requires different sets of skills, knowledge, supplies and equipment to provide certain packages of care including the provision of RH-HIV integrated services. The provision of these services is also depended on the availability of certain cadres of health care workers at the six levels of service delivery [4]. Thus, clients or patients navigate between these levels of care depending on the type of health services they are seeking and whether the key inputs such as skills, supplies are available in certain health facilities or not.
Between November 2005 and March 2007, a project that sought to test the feasibility of implementing two models (testing and referral models) for integrating counseling and testing for HIV into family planning services was carried out in 23 health facilities in the Central region of Kenya [19]. The findings showed that implementing both models was feasible and acceptable to clients and providers. A major limitation of the 2005-2007 integration project was that there was no mechanism of linking family planning clients who had been tested and found to be positive to treatment and care facilities on site or offsite. The gap meant that program staff could not tell or establish the actual number of clients who were linked to treatment and care services including anti-retroviral therapy (ART). Besides, overall evidence on effectiveness of linking family planning clients who test HIV positive to comprehensive care centers regarding such outcomes as uptake of care and support services as well as initiation of clients to antiretroviral therapy for those eligible is scanty and inconclusive.

In order to address the gap, the project staff who were involved in the design and implementation of the 2005-2007 FP/HIV integration project developed and tested (two years later) a modified approach for linking HIV positive family planning clients to comprehensive care centers that offer HIV treatment and care services. The key question addressed by the modified project was: "what is the most effective mechanism for linking family planning clients found to be HIV positive to treatment and are services?" [20]. The overall objective of the modified project was to develop a referral framework for linking family planning clients who test HIV positive to treatment and care centers in selected health facilities. The specific objectives of the project were to assess: (i) facility preparedness in terms of key health providers, FP commodities, STI drugs, infrastructure and HIV test kits and reagents, (ii) the acceptability of the proposed referral framework to providers, and (iii) the effectiveness of the referral framework in terms of increasing the number of eligible HIV-positive FP clients received at HIV care and treatment services. This paper examines the acceptability and effectiveness of linking HIV-positive family planning clients to treatment and care services among health care providers under the project.

\section{Materials and Methods}

\section{Project Design}

The project design involved a pre- and post-intervention data collection without a comparison group. Pre-intervention (baseline) data collection involved a review of records for a period of 6 months from August 2009 to March 2009. After baseline data collection, we began implementing intervention activities for a period of 6 months (between December 2009 and May 2010) in selected sites in Nyeri County and Thika District of Kiambu County in the Central region of Kenya. At the end of the intervention period, a post-intervention data collection exercise (endline survey) was conducted during the months of June and July in 2010. A total of six intervention sites were purposefully selected for inclusion in the study from the original 23 project sites that were already offering integrated FP/HIV services. A key criterion for inclusion of a health facility in the study was availability of a functional comprehensive care centre as part of its routine operations. The six health facilities selected were: the Provincial General Hospital in Nyeri, Warazo Sub-District Hospital, Thika District Hospital, Gatundu District Hospital, Ruiru Sub-District Hospital and Kirwara Sub-District Hospital. Table 1 presents a summary of the Family Planning workload at the project sites (Table 1).

Two hypotheses guided the evaluation of the project: those HIV- 


\begin{tabular}{|l|l|l|}
\hline \multirow{2}{*}{ Facility } & \multicolumn{2}{l|}{$\begin{array}{l}\text { Number of clients attending family planning } \\
\text { services }\end{array}$} \\
\cline { 2 - 3 } & $\begin{array}{l}\text { Baseline (August } \\
2009-\text { March 2009) }\end{array}$ & $\begin{array}{l}\text { Endline (December } \\
2009-\text { May 2010) }\end{array}$ \\
\hline Nyeri Provincial General Hospital & 2,803 & 2,753 \\
\hline Thika District Hospital & 6,104 & 6,203 \\
\hline Gatundu District Hospital & 3,623 & 4,229 \\
\hline Ruiru Sub- District Hospital & 2,624 & 2,367 \\
\hline Kirwara Sub- District Hospital & 1,847 & 1,708 \\
\hline Warazo Health Center & 1,254 & 1,998 \\
\hline Total & 18,255 & 17,550 \\
\hline
\end{tabular}

Table 1: Workload data in family planning clinics pre- and post-intervention period.

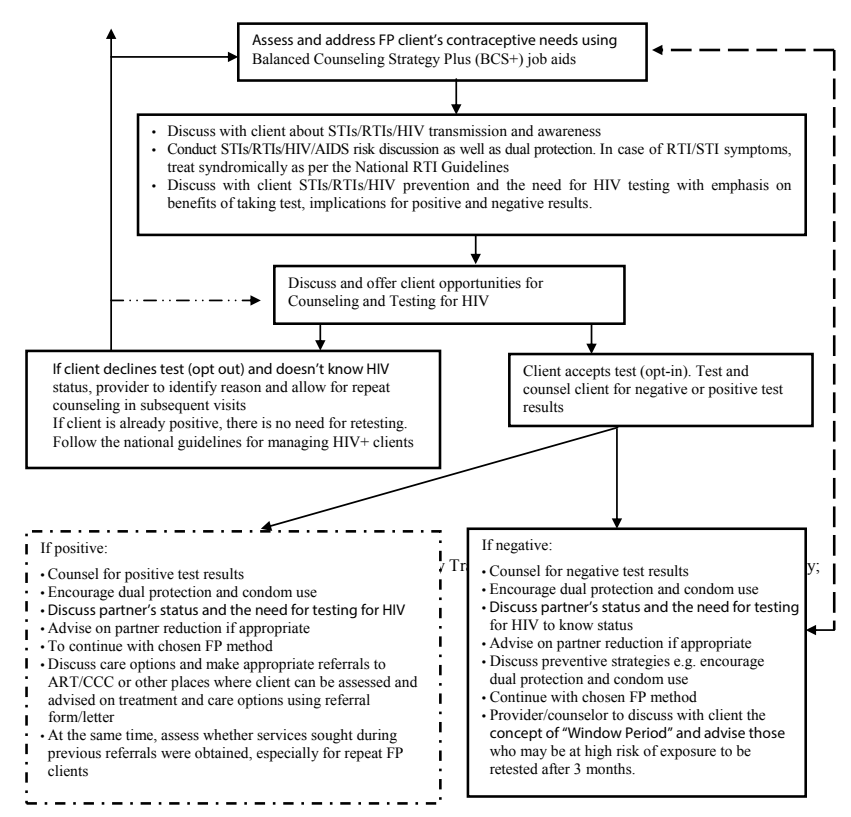

Figure 1: Modified Protocol for counseling and testing clients for HIV in FP settings.

positive FP clients in intervention clinics were more likely to receive referral to ART services after the intervention than before, and that HIV positive FP clients were more likely to visit the comprehensive care centers (CCCs) after the intervention had been introduced than before. The project obtained ethical approval from the Population Council's Institutional Review Board (IRB) and Kenya Medical Research Institute (KEMRI) Ethical Review Committee (ERC) before starting.

\section{Intervention development, implementation and monitoring}

The interventions were conceptualized around a modified algorithm for linking FP clients who test HIV-positive to care and treatment services (Figure 1). The algorithm was developed during the design phase of the project to guide health providers in counseling and testing clients wishing to know their HIV status. It was designed for use in facilities where the FP provider carries out the HIV test during the FP consultation, or at least on the same day and at the same facility. This algorithm does not apply to clients being referred to other facilities to be tested for HIV, since the person doing the HIV test is the one who would have responsibility for linking an HIV-positive client to the CCC. The interventions involved five activities that were implemented over a period of six months (December 2009 - May 2010).
The first activity involved strengthening referral system for HIVpositive FP clients. Under this activity, a modified, strengthened referral system for HIV-positive FP clients was introduced in the six sites following the steps described in Box I.

\section{Box 1: Steps followed in conducting referrals of HIV-positive FP clients}

1. Care options were discussed between a client and provider and appropriate referrals made using the referral form (See Annex 1) which was designed to capture information on client background characteristics, name of facilities conducting referrals and receiving clients, reasons for referral and appropriate comments

2. Client told that the referral form is in triplicate. Original copy to be given to the client to take to the CCC (care and treatment centre of the receiving facility), duplicate copy is kept by the District Reproductive Health Coordinator (project staff) and triplicate retained in the referral book at referring facility/ clinic. (However, during the dissemination of study findings, the $\mathrm{MOH}$ recommended that as referral practices become institutionalized, there will be no need for the triplicate copies. Duplicate copies will be sufficient. The original copy will be given to the client to take to the care and treatment centre while the duplicate copy will be retained in the referral book in the facility initiating the referral).

3. Once client arrives at the receiving facility, she/he is told to hand in the original form to the nursing officer in charge of CCC (or the officer on duty) who is expected to fill the referral forms, sign and file them in a designated referrals folder.

4. Client counseled on what to expect in CCCs including discussion on the types of investigations or assessments the client was likely to go through e.g. testing and counseling, expected cost of treatment (especially if the facility is private) among other pieces of information.

5. Client assured that the management of her or his condition is dependent on the outcome of the physical clinical assessment and laboratory evaluation of her or his own immunity using CD4 count.

6. Client told that regardless of his/her state of immunity, everyone starts on Septrin (cotrimoxazole) to prevent opportunistic infections.

The second activity involved review of training materials and job aids. Existing training and participant manuals for integrating counseling and testing for HIV into family planning services were reviewed and updated to include the component on screening for HIV, knowing status, and referrals.

The third activity focused on training service providers on updated tools. Providers at the six sites were updated on the modified referral system, revised training materials and on the referral tool (Annex 1). A mentorship approach was used in updating the providers' knowledge and skills required to effectively link HIV-positive FP clients to ART/STI services. Mentorship is a system of practical training and consultation that fosters ongoing professional development to yield sustainable highquality HIV/RH/FP services. Mentoring of less experienced individuals (mentees) by experienced staff (mentors) was an essential element of all health care learning in the pilot facilities. The need for mentorship arose from the desire to transform the existing didactic training, which has not always been successful in translating the acquired knowledge into practice, especially when conducted outside of the health facility settings.

The fourth activity focused on strengthening the referral system, which involved two major interventions: (i) strengthening the management information system; and (ii) introducing a referral tool. FP registers at each facility provided the main source of information on the number of clients referred to ART services. Although the FP registers have information on clients counseled and tested for HIV, there was no indication whether those who tested positive were referred to the CCCs. The project therefore trained providers to indicate whether the clients were referred to the CCCs in the remarks column in the FP registers. In addition, the project developed a referral form (Annex 
1) in collaboration with DRH and NASCOP. This was then pre-tested before being shared with service providers in the field. Each booklet had 50 copies of the referral forms in triplicate - the client was given the original copy to take to the receiving clinic/site and the duplicate copy was retained in the facility making the referral. The triplicate copy was retained in the booklet. The receiving site kept a file of all the received referral forms.

The fifth activity involved strengthening of supervision activities and monitoring of interventions. Members of the Provincial Health Management Team (PHMT) and District Health Management Team (DHMT) made monthly supervisory visits to the facilities to monitor the implementation of the activities. They used a structured supervisory tool to collect information on background data, services offered in the facility, staffing profile, staffing and training updates in the last 24 months, availability of RH and HIV commodities in the previous month, status of equipment, supervision for quality improvement, monitoring and evaluation, referral and communication system and recommendations for improvement. In addition, a team of staff from DRH, NASCOP and Population Council conducted quarterly supervisory visits to assess referral practices for HIV-positive clients at facilities making referrals and those receiving referred clients.

\section{Data collection and analysis}

Data collection involved facility assessment, provider interviews, and record reviews. Facility assessment involved a systematic inventory and interviews with facility in-charges using a checklist to determine the availability of staff, equipment and supplies. The purpose was to determine the readiness of the facilities to link HIV-positive FP clients to ART. Interviews with providers, on the other hand, were conducted using a structured questionnaire that captured information on training in, knowledge of, and experience with counseling and referral as well as the national guidelines on the same. The record reviews involved extracting data from client records using a checklist that was developed based on the national guidelines for managing HIV-positive clients in Kenya. In particular, information was extracted on source of referral, whether client went through pre-ART counseling, appropriate records that constitute standard care for HIV-positive clients, whether the records are appropriately filled, and whether all the necessary procedures were followed and documented.

Table 2 presents the sample sizes achieved at both baseline and endline for the various components of the study (facility assessment, records review, and providers interviews). Besides the review of records in each of the facilities selected, at least one interview with the officer-in charge and four providers were carried out. Analysis of the data entails descriptive statistics including frequencies, percentages and scores with significant tests of means and proportions to determine if there were any significant differences between baseline and endline in the indicators of quality of care.

\section{Results}

\section{Characteristics of clients whose records were reviewed}

At both baseline and endline, the mean and median age of clients from the records was 33 years, with the oldest client being 60 years and the youngest 18 years old. Repeat and new FP clients constituted $97 \%$ and $3 \%$ of the total clients respectively at baseline while at endline repeat clients constituted $94 \%$ and new clients $6 \%$. The majority of clients were married and in monogamous relationships (63\% at both baseline and endline). Within the CCCs, over 97\% of the records had CD4 values with $61 \%$ of the values being below 350 while $39 \%$ were above this cutoff range in both baseline and end line surveys.

Facility preparedness: Facility preparedness was assessed by examining the availability of the following cadres of health providers and supplies:

- Staff (medical officers, nurses, clinical officers, pharmacists and pharmaceutical technologists);

- Registers and cards: 6 items (partner notification card, client card, FP, pre-ART, ART, and antiretroviral drugs registers);

- FP commodities: 6 items (combined oral pill, depo provera, implants, male condoms, and female condoms);

- STI drugs: 5 items (drugs for management of urethral discharge, genital ulcers, ophthalmia neonatorum, vaginal discharge/ pruritus, and lower abdominal pain);

- HIV test kits and reagents: 6 items (Determine, Bioline, Unigold, laboratory reagents for urinalysis, full haemogram reagents, and infection prevention items); and

- Infrastructure: 6 items (availability of directory for use in referring clients to the CCCs and other STI services, referral guidelines; a register for documenting referral practices; client/patient tracer card; mechanisms for reviewing referral decisions/ arrangements e.g. through monthly meetings; and feedback mechanisms between referring and receiving facilities).

Table 3 presents the distribution of key indicators of facility preparedness to support referral of HIV-positive family planning clients at baseline at maternal and child health $(\mathrm{MCH}) / \mathrm{FP}$ and CCC units. The results show that in terms of staffing; only three of the six facilities had the minimum number of staff necessary to support referral and linkage of HIV-positive FP clients to treatment and care. In addition, only two of the six facilities had all the essential registers and tracer/client cards to support referral of HIV-positive clients to treatment and care at baseline. With respect to the availability of FP commodities, none of the facilities had all the six FP commodities (combined oral pill, depo provera, implants, IUCD, male condoms, and female condoms) and half had only four of the commodities. Female

\begin{tabular}{|c|c|c|c|c|c|c|c|c|}
\hline Study component & Timing /Phase & Nyeri PGH & Thika DH & Gatundu DH & Ruiru SDH & Kirwara DH & Warazo HC & Total Sample \\
\hline \multirow{2}{*}{$\begin{array}{l}\text { FP Client Records Review in } \\
\text { CCCs }\end{array}$} & Baseline & 120 & 120 & 120 & 37 & 36 & 21 & 454 \\
\hline & Endline & 87 & 150 & 86 & 99 & 37 & 28 & 487 \\
\hline \multirow{2}{*}{$\begin{array}{l}\text { Provider } \\
\text { Interviews }\end{array}$} & Baseline & 4 & 4 & 4 & 4 & 4 & 4 & 24 \\
\hline & Endline & 4 & 4 & 4 & 4 & 4 & 4 & 24 \\
\hline \multirow{2}{*}{ Facility Assessment } & Baseline & 1 & 1 & 1 & 1 & 1 & 1 & 6 \\
\hline & Endline & 1 & 1 & 1 & 1 & 1 & 1 & 6 \\
\hline
\end{tabular}

Notes: PGH: Provincial General Hospital; DH: District Hospital; SDH: Sub-District Hospital; HC: Health Center; FP: Family Planning; CCCs: Comprehensive Care Centers Table 2: Sample sizes achieved by type of survey conducted at baseline and endline by facility. 


\begin{tabular}{|c|c|c|c|c|c|c|c|}
\hline Indicators & $\begin{array}{l}\text { Thika } \\
\text { DH }\end{array}$ & $\begin{array}{l}\text { Ruiru } \\
\text { SDH }\end{array}$ & $\begin{array}{c}\text { Gatundu } \\
\text { DH }\end{array}$ & $\begin{array}{l}\text { Nyeri } \\
\text { PGH }\end{array}$ & $\begin{array}{c}\text { Kirwara } \\
\text { SDH }\end{array}$ & $\begin{array}{c}\text { Warazo } \\
\text { HC }\end{array}$ & Total \\
\hline \multicolumn{8}{|l|}{ Staff availability (number) } \\
\hline Medical officers & 5 & 0 & 1 & 4 & 0 & 0 & \\
\hline Nurses & 23 & 7 & 4 & 12 & 4 & 2 & \\
\hline Clinical officers & 3 & 1 & 2 & 3 & 2 & 0 & \\
\hline Pharmacists/PT & 3 & 0 & 0 & 0 & 0 & 0 & \\
\hline Availability of registers and cards (score on 6 items) & 5 & 6 & 5 & 5 & 4 & 6 & $31 / 36$ \\
\hline Availability of key FP commodities (score on 6 items) & 3 & 4 & 3 & 4 & 4 & 3 & $21 / 36$ \\
\hline Availability of STI drugs (score on 5 items) & 5 & 2 & 2 & 0 & 5 & 2 & $16 / 30$ \\
\hline Availability of HIV test kits and reagents (score on 6 items) & 6 & 6 & 5 & 2 & 6 & 4 & $29 / 36$ \\
\hline Availability of infrastructure (score on 6 items) & 1 & 4 & 1 & 3 & 0 & 0 & $9 / 36$ \\
\hline
\end{tabular}

Notes: PGH: Provincial General Hospital; DH: District Hospital; SDH: Sub-District Hospital; HC: Health Center; FP: family planning; MCH: maternal and child health; CCC: comprehensive care center; STIs: sexually transmitted infections; PT: pharmaceutical technologist.

Table 3: Distribution of key indicators of facility preparedness to support referral of HIV-positive family planning clients at baseline at MCH/FP and CCC units.

Indicators

Proportion of records showing WHO clinical staging for HIVIAIDS had been done

Proportion of records showing CD4+ values

Proportion of records showing whether clients are on ARVs

Proportion of records showing that the client had just started HAART (within 6 months)

Proportion of records that had the necessary documents filled correctly

Number of cases

\begin{tabular}{|c|c|}
\hline Baseline (\%) & Endline(\%) \\
\hline 90 & 89 \\
\hline 97 & 98 \\
\hline 34 & $49^{*}$ \\
\hline 8 & $25^{*}$ \\
\hline 15 & $28^{*}$ \\
\hline 454 & 487 \\
\hline
\end{tabular}

Notes: ${ }^{a} \mathrm{WHO}$ staging system is a simplified AIDS staging system that is based on 4 groups of clinical conditions that have prognostic significance and therefore constitute stages, plus an assessment of physical activity performance expressed as a 4-point score; Patients are classified according to the highest stage recorded for either clinical condition or physical activity. Patients are asymptomatic; mildly symptomatic; moderately symptomatic; severely symptomatic. Clinical Stage 1 (asymptomatic, normal activity), Clinical Stage 2 (Unintentional weight loss $<10 \%$ body weight), Clinical Stage 3(Unintentional weight loss > 10\% body weight, Chronic diarrhea > 1 month, prolonged fever > 1 month (constant or intermittent), Clinical Stage 4 (HIV wasting syndrome, severe opportunistic infection and severely ill); CCCs: Comprehensive Care Centers; WHO: World Health Organization; ARVs: antiretrovirals; HAART: Highly Active Antiretroviral Therapy; ${ }^{*}<<0.05$.

Table 4: Distribution of key indicators of quality of care within the CCCs.

condoms and implants were the least available FP methods. Only two facilities had all the required STI drugs in the CH/FP and CCC units at baseline and endline. At Nyeri PGH, the drugs were available at the pharmacy, but none at the MCH/FP and CCC units. The results in Table 3 further show that only two of the facilities scored three or higher on the indicators of infrastructure availability.

Acceptability of referral by providers: Two indicators measured acceptability of referral of HIV-positive FP clients to treatment and care among providers: the proportion of providers reporting having a directory of facilities and their contact details where HIV-positive clients could be referred and the proportion of providers reporting discussing referral issues during routine management meetings. The results show that the proportion of providers who used a directory for referral rose from $8 \%$ at baseline to $58 \%$ at endline $(p<0.01)$ while the proportion of providers who discussed referral of HIV-positive FP clients improved from $71 \%$ at baseline to $96 \%$ at the endline $(p<0.05)$. The use of the directory at baseline was due to the fact that another organization, the International Center for AIDS Care and Treatment Programs (ICAP) had introduced it earlier at Thika District Hospital.

Quality of care: Results from the records review show that there was a significant improvement at endline in the provision of ARVs, HAART and proper documentation of records, compared to the situation at the baseline (Table 4). Specifically there were significant improvements in the proportion of records showing whether clients were on ARVs, that the client had just started HAART (within 6 months) and of records that had the necessary documents filled correctly at endline compared to the baseline.

Effectiveness of referral: Effectiveness of referral of HIV-positive
FP clients to treatment and care was determined from information from the record reviews. The results show that initially, there was no referral of HIV-positive FP clients to CCCs. However, during the intervention period, most of the HIV-positive FP clients received at CCCs (93\%) were from within the MCH/FP unit of the same facility indicating that the referral system was effective (Table 5). These results also demonstrate the extent to which on-site referrals between the various sections or units were effective as opposed to off-site referrals between the various facilities. Being a pilot project, off-site referrals were expected to be minimal or negligible.

\section{Discussion}

One of the major findings of the study is that although most facilities were not adequately prepared in terms of infrastructure to support linking HIV-positive family planning clients to treatment and care services, there was significant improvement in provider acceptance of referral practices after than before the interventions. In addition, the referral system was very effective as most of the HIV-positive FP clients received at CCCs were from within the MCH/FP unit of the same facility. It is also worth noting that by the end of the study period, most of the facilities had the required supplies to ensure effective linkage of HIV-positive FP clients to treatment and care. It could be that facility preparedness and the performance of the providers with respect to referral were enhanced by the supervisory visits that were conducted by members of PHMT, DHMT, DRH and NASCOP. It was likely that during such visits, providers identified areas of need and members of the supervisory teams responded to those needs in a timely manner to ensure the success of the project. The findings further support the arguments for integrating $\mathrm{RH}$ and HIV services in terms of enhancing clients' access to care [4]. 


\begin{tabular}{|c|c|c|c|c|}
\hline Health facility & $\begin{array}{l}\text { Number of HIV- } \\
\text { positive FP clients }\end{array}$ & $\begin{array}{l}\text { Number of HIV-positive FP clients } \\
\text { received at CCC from within facility* }\end{array}$ & $\begin{array}{l}\text { Proportion of HIV-positive FP clients } \\
\text { received at CCC from within facility) }\end{array}$ & $\begin{array}{c}\text { Number of HIV-positive FP clients received } \\
\text { at CCC from other facilities** }\end{array}$ \\
\hline Nyeri PGH & 3 & 3 & 100 & 10 \\
\hline Thika DH & 9 & 9 & 100 & 9 \\
\hline Gatundu DH & 10 & 9 & 90 & 8 \\
\hline Ruiru SDH & 40 & 39 & 98 & 3 \\
\hline Kirwara SDH & 4 & 2 & 50 & 0 \\
\hline Warazo HC & 5 & 3 & 60 & 0 \\
\hline Total & 71 & 66 & 93 & 30 \\
\hline \multicolumn{5}{|c|}{$\begin{array}{l}\text { Number of HIV-positive FP clients received at CCC from within facility shows the extent to which onsite referrals between } \\
\text { the various sections or units are being implemented. } \\
\text { ** Number of HIV-positive FP clients received at CCC from other facilities shows the extent to which off-site referrals between } \\
\text { the various facilities are being implemented. }\end{array}$} \\
\hline
\end{tabular}

Notes: PGH: Provincial General Hospital; DH: District Hospital; SDH: Sub-District Hospital; HC: Health Center; FP: family planning; CCC: comprehensive Care Center.

Table 5: Distribution of HIV-positive family planning clients referred and received at comprehensive care centers from within the facility, January-June 2010.

The study findings have important lessons for programs aimed at linking HIV-positive FP clients to treatment and care. First, minimal preparedness on the part of the facilities may not be a serious limiting factor for effective referral if mechanisms for support supervision are put in place. A key challenge, however, is ensuring the sustainability of regular supervisory visits to facilities given the financial implications of such visits. Second, the modified algorithm for linking HIV-positive family planning clients to treatment and care services was designed for use in facilities where HIV testing is carried out by FP provider during FP consultation, or at least on the same day and at the same facility. The algorithm does not apply to clients being referred to other facilities to be tested for HIV since the person doing the HIV test is the one who would have responsibility for linking an HIV-positive client to treatment and care. This implies that providers should not only be fully equipped with appropriate skills for providing RH services including FP services but also have skills for counseling and testing for HIV. Following the launch of the provider initiated testing and counseling (PITC) policy by the Government of Kenya in 2008, the proportion of clients attending FP/RH clinics who knew their status increased tremendously [21]. In addition, the proportion of clients who either preferred to be referred to other health facilities to be tested for HIV or to access care and treatment in facilities of their choice increased [21]. The implication is that the different scenarios require different algorithms.

The findings might be affected by the limitations of the study. For instance, the acceptability and effectiveness of the referral mechanism could be a function of the small number of facilities that participated in the project. It is more effective in terms of cost and time to monitor a small than a large number of facilities. In addition, the findings could be a result of the Hawthorne effect, that is, providers could have improved their performance because they knew they were participating in a study and therefore being observed [22]. To the extent that this was the case, the impact of the interventions in the present study would be an over-estimate. The other limitation stems from the differences in the participating health facilities in terms of level as defined by the Kenya Essential Package for Health [23]. In particular, two of the facilities (Thika District Hospital and Nyeri Provincial General Hospital) are Level 5 referral health facilities; Gatundu District Hospital is Level 4 while Ruiru Sub-District Hospital, Kirwara Sub-District Hospital, and Warazo Health Center are Level 3 facilities that essentially function as health centers. The implication is that referrals at Levels 4 and 5 facilities are likely to be internal, hence easy to trace while the lower level facilities are likely to refer clients to higher level ones thereby complicating client tracing. Another limitation of the study had to do with extracting information from some records that had incomplete or partially filled data. Examples of these records included official $\mathrm{MOH}$ cards and registers that are used in enrolling patients for treatment and care at the Comprehensive Care Centres (CCCs).

Despite the limitations, the findings of the study show that referral and linking of HIV-positive FP clients to treatment and care services is acceptable and effective even with minimal levels of preparedness on the part of facilities. In particular, the use of the referral forms and a directory of facilities coupled with support supervision is an effective mechanism for ensuring effective referrals and linkage. The fact that the tools were introduced in facilities with minimal infrastructure requirements further suggests that they can easily be scaled up for use nationally in addition to being adopted for use in other departments including antenatal and post-natal care clinics as a tool for referring HIV-positive clients to appropriate care and treatment.

\section{Acknowledgments}

The study that provided the data for this paper was funded by the United States Agency for International Development (USAID)/Kenya Mission through cooperative agreement No. 623-A-00-09-00001-00 between the Mission and the Population Council. The study was implemented under APHIA II Operations Research Project/ Population Council in collaboration with the National HIVIAIDS and STDs Contro Programme (NASCOP) and the Division of Reproductive Health, Ministry of Health Kenya. The paper benefitted from valuable comments from anonymous reviewers. However, the opinions expressed in the paper are those of the authors and do not necessarily reflect the views of the funding or implementing agencies.

\section{References}

1. Liambila W (2012) Fact sheet: Reproductive health-HIV integration activities in Kenya.

2. Karen G, Foreit K, Hardee K, Agarwal K (2002) When does it make sense to consider integrating STI and HIV services with family planning services? International Family Planning Perspectives 28: 105-107.

3. UNAIDS (2011) UNAIDS Terminology Guidelines. Geneva: UNAIDS

4. Republic of Kenya (2012) Minimum Package for RH-HIV Integration in Kenya Nairobi: Ministry of Health/ National AIDS/STI Control Programme and Division of Reproductive Health.

5. Caldwell JC, Caldwell $P(2002)$ Is integration the answer for Africa? International Family Planning Perspectives 28:108-110.

6. Reynolds HW, Janowitz B, Wilcher R, Cates W (2008) Contraception to prevent HIV-positive births:current contribution and potential cost-savings in PEPFAR countries. Sexually Transm Infect 84: ii49-ii53.

7. Sweat MD, O'Reilly KR, Schmid GP, Denison J, de Zoysa I (2004) Costeffectiveness of nevirapine to prevent mother-to-child HIV transmission in eight African countries. AIDS 18:1661-1671.

8. Halperin DT, Stover J, Reynolds HW (2009) Benefits and costs of expanding access to family planning programs to women living with HIV. AIDS: S123-S130. 
Citation: Liambila W, Obare F, Birungi H, Kuria SN, Muia RW, et al. (2013) Acceptability and Effectiveness of Linking HIV-Positive Family Planning Clients to Treatment and Care Services among Health Care Providers in Kenya. J AIDS Clin Res 4: 232. doi:10.4172/2155-6113.1000232

Page 7 of 7

9. Reynolds HW, Steiner MJ, Cates W Jr (2005) Contraception's proved potential to fight HIV. Sexually Transmitted Infections 81:184-185.

10. Reynolds H, Janowitz B, Homan R, Johnson L (2006) The value of contraception to prevent perinatal HIV transmission. Sexually Transmitted Diseases 33: 350 356

11. Askew I, Berer M (2003) The contribution of sexual and reproductive health services to the fight against HIVIAIDS: a review. Reprod Health Matters 11: 5173.

12. Lush L, Cleland J, Walt G, Mayhew S. (1999) Integrating reproductive health: myth and ideology. Bull World Health Organ 77: 771- 777.

13. Homsy J, Bunnell R, Moore D, King R, Malamba S, Nakityo R, et al. (2009) Reproductive intentions and outcomes among women on antiretroviral therapy in rural Uganda: a prospective cohort study. PLoS One 4: e4149.

14. Wilcher R, Cates W, Gregson S (2009) Family planning and HIV: strange bedfellows no longer. AIDS 23: S1-S6.

15. Church K, Mayhew SH (2009) Integration of STI and HIV Prevention, Care, and Treatment into Family Planning Services: A Review of the Literature. Stud Fam Plann 40: 171-186.

16. Republic of Kenya (2012) Minimum Package for RH-HIV Integration in Kenya Published by the National AIDS and STI Control Programme and the Division of Reproductive Health, Ministry of Health Headquarters, Nairobi - Kenya.
17. Askew I, Maggwa NB (2002) Integration of STI Prevention and Management with Family Planning and Antenatal Care in sub-Saharan Africa-What More Do We Need to Know? International Family Planning Perspectives 28: 77-86.

18. Family Health International (2006) Integrating Family Planning into HIV Voluntary Counseling and Testing Services in Kenya: Progress to Date and Lessons Learned. Family Health International, Research Triangle Park, USA

19. Liambila W, Askew I, Mwangi J, Ayisi R, Kibaru J, et al. (2009) Feasibility and effectiveness of integrating provider initiated testing and counseling within family planning services in Kenya. AIDS 23: S115-S121.

20. Liambila W, Obare F, Birungi H, Wayua MR, Maina J, et al. (2011) Linking HIV - Positive Family Planning Clients to Treatment and Care Services in Kenya. Nairobi: APHIA II OR Project Report / Population Council.

21. Ministry of Public Health and Sanitation (2008) National Guidelines for HIV Counselling and Testing in Kenya. Ministry of Public Health and Sanitation/ National AIDS and STD Control Programme.

22. Mugenda OM, Mugenda AG (1999) Research Methods: Quantitative and Qualitative Approaches. Nairobi: Acts Press.

23. Ministry of health (2006) Taking the Kenya Essential Package for Health to the Community: A Strategy for Delivery of Level One Services. Nairobi: Republic of Kenya/Ministry of Health, Kenya. 